\title{
CHARACTERIZING COSMIC-RAY PROPAGATION IN MASSIVE STAR-FORMING REGIONS: THE CASE OF 30 DORADUS AND THE LARGE MAGELLANIC CLOUD
}

\author{
E. J. Murphy ${ }^{1}$, T. A. Porter ${ }^{2,3}$, I. V. Moskalenko ${ }^{2,3}$, G. Helou $^{4}$, and A. W. Strong ${ }^{5}$ \\ ${ }^{1}$ Observatories of the Carnegie Institution for Science, 813 Santa Barbara Street, Pasadena, CA 91101, USA; emurphy @ obs.carnegiescience.edu \\ ${ }^{2}$ Hansen Experimental Physics Laboratory, Stanford University, Stanford, CA 94305, USA; tporter@ stanford.edu, imos@ stanford.edu \\ ${ }^{3}$ Kavli Institute for Particle Astrophysics and Cosmology, Stanford University, Stanford, CA 94305, USA \\ ${ }^{4}$ California Institute of Technology, MC 100-22, Pasadena, CA 91125, USA; gxh@ipac.caltech.edu \\ ${ }^{5}$ Max-Planck-Institut für extraterrestrische Physik, Postfach 1312, D-85741 Garching, Germany; aws@mpe.mpg.de \\ Received 2012 January 24; accepted 2012 March 7; published 2012 April 24
}

\begin{abstract}
Using infrared, radio, and $\gamma$-ray data, we investigate the propagation characteristics of cosmic-ray (CR) electrons and nuclei in the 30 Doradus (30 Dor) star-forming region in the Large Magellanic Cloud (LMC) using a phenomenological model based on the radio-far-infrared correlation within galaxies. Employing a correlation analysis, we derive an average propagation length of $\sim 100-140 \mathrm{pc}$ for $\sim 3 \mathrm{GeV}$ CR electrons resident in 30 Dor from consideration of the radio and infrared data. Assuming that the observed $\gamma$-ray emission toward 30 Dor is associated with the star-forming region, and applying the same methodology to the infrared and $\gamma$-ray data, we estimate $\mathrm{a} \sim 20 \mathrm{GeV}$ propagation length of $200-320 \mathrm{pc}$ for the CR nuclei. This is approximately twice as large as for $\sim 3 \mathrm{GeV}$ CR electrons, corresponding to a spatial diffusion coefficient that is $\sim 4$ times higher, scaling as $(R / \mathrm{GV})^{\delta}$ with $\delta \approx 0.7-0.8$ depending on the smearing kernel used in the correlation analysis. This value is in agreement with the results found by extending the correlation analysis to include $\sim 70 \mathrm{GeV} \mathrm{CR}$ nuclei traced by the $3-10 \mathrm{GeV}$ $\gamma$-ray data $(\delta \approx 0.66 \pm 0.23)$. Using the mean age of the stellar populations in 30 Dor and the results from our correlation analysis, we estimate a diffusion coefficient $D_{R} \approx(0.9-1.0) \times 10^{27}(R / \mathrm{GV})^{0.7} \mathrm{~cm}^{2} \mathrm{~s}^{-1}$. We compare the values of the CR electron propagation length and surface brightness for 30 Dor and the LMC as a whole with those of entire disk galaxies. We find that the trend of decreasing average CR propagation distance with increasing disk-averaged star formation activity holds for the LMC, and extends down to single star-forming regions, at least for the case of 30 Dor.
\end{abstract}

Key words: cosmic rays - galaxies: individual (LMC) - gamma rays: galaxies - H II regions - infrared: galaxies radio continuum: galaxies - stars: formation

\section{INTRODUCTION}

Cosmic rays (CRs) are a dynamically important component of the interstellar medium (ISM) in galaxies. Yet their role in shaping galaxy evolution is currently unclear due to the difficulties in characterizing their origin and propagation in the ISM (e.g., Strong et al. 2007). The energy density of CRs is comparable to that of magnetic fields, as well as the radiation fields and turbulent motions of the interstellar gas in galaxies. Together, CRs and large-scale magnetic fields comprise a relativistic plasma whose interactions with the interstellar gas shape the overall chemistry and heating of the ISM (e.g., Boulares \& Cox 1990; Ferrière 2001; Cox 2005), and may also play a significant role regulating star formation processes (e.g., Socrates et al. 2008; Papadopoulos 2010; Papadopoulos et al. 2011).

The diffuse emissions from the radio to high-energy $\gamma$ rays $(>100 \mathrm{MeV})$, produced by various interactions between CRs and the interstellar gas, radiation, and magnetic fields, are the best way to characterize the physics of CRs throughout most of the Milky Way and other galaxies. For example, the CR lepton component can be probed by radio, X-ray, and $\gamma$-ray observations. The radio emission arising from the injection of CR electrons from supernova remnants trace these particles as they propagate through large-scale magnetic fields and lose energy from synchrotron radiation. Similarly, the same CR electrons inverse Compton (IC) scatter off the interstellar radiation field (ISRF) and cosmic microwave background, yielding observable emissions from X-ray to $\gamma$-ray energies
(Porter et al. 2008). High-energy $\gamma$ rays are particularly useful because this energy range gives access to the dominant hadronic component in CRs via the observation of $\gamma$ rays from the decay of neutral pions produced by inelastic collisions between CR nuclei and the interstellar gas (Pollack \& Fazio 1963).

Externally viewed galaxies have the advantage of mitigating line-of-sight confusion that hampers interpretation of the diffuse emissions of the Milky Way. While such observations can be used to determine the present distribution of CRs, understanding their propagation history remains difficult without knowledge of the initial distribution of CR sources. Using the tight, empirical correlation between the far-infrared (FIR) and (predominantly) non-thermal radio continuum emission from galaxies (de Jong et al. 1985; Helou et al. 1985), a number of studies have attempted to characterize the propagation of CR electrons in external galaxies (e.g., Marsh \& Helou 1998; Murphy et al. 2006a, 2008). The underlying physics relating the radio and FIR emission from galaxies lies in the process of massive star formation: young massive stars are the dominant sources of dust heating, and end their lives as supernovae whose remnants presumably accelerate and inject CR electrons into the ISM where they produce diffuse synchrotron emission. It was hypothesized that the radio image of a galaxy should resemble a smoothed version of its infrared image, because the mean free path of dust-heating photons $(\sim 100 \mathrm{pc})$ is significantly shorter than the typical diffusion length of CR electrons $(\sim 1-2 \mathrm{kpc}$; Bicay \& Helou 1990). This phenomenology is supported by studies within nearby galaxies at kiloparsec (e.g., Marsh \& Helou 1998) and few-hundred-parsec (e.g., Murphy et al. 
2006a) scales, as well as on scales of $\gtrsim 50$ pc in the Large Magellanic Cloud (LMC; Hughes et al. 2006). Investigations on sub-kiloparsec scales within a sample of nearby spirals have even shown a dependence of the typical propagation length of CR electrons on star formation activity arising from the predominant youth of $\mathrm{CR}$ electron populations in galaxies with enhanced disk-averaged star formation rates (SFRs; Murphy et al. 2006b, 2008).

Recently, using 11 months of data, the LMC was detected by the Fermi Large Area Telescope (LAT; Abdo et al. 2010b) at high significance with the resolved $\gamma$-ray emission showing very little correlation with the large-scale distribution of the gas column density. If CRs freely diffuse in the ISM of the LMC, as they appear to in the Milky Way, the $\gamma$-ray emission should be correlated with the distribution of gas, which is predominantly neutral hydrogen and helium in the LMC (Staveley-Smith et al. 2003). Instead, the observed $\gamma$-ray emission for the LMC is more strongly correlated with tracers of massive star-forming regions. Employing the multifrequency diffuse emissions from radio to $\gamma$-ray energies, we can investigate for the first time the propagation of both $\mathrm{CR}$ electrons and nuclei associated with a star-forming region in an externally resolved galaxy, 30 Doradus (30 Dor) in the LMC.

\section{DATA AND ANALYSIS}

We compiled archival infrared and radio data, together with 32 months of $\gamma$-ray data from Fermi-LAT for our analysis. We assume a distance to 30 Dor of $50 \mathrm{kpc}$ throughout this analysis. ${ }^{6}$

\subsection{Spitzer Infrared Data}

Spitzer imaging of the LMC was carried out as part of the Surveying the Agents of a Galaxy's Evolution (SAGE; Meixner et al. 2006) legacy program. The observations covered an area of $\sim 7^{\circ} \times 7^{\circ}$ over the LMC in all $\operatorname{IRAC}(3.6,4.5,5.8$, and $8 \mu \mathrm{m})$ and MIPS $(24,70$, and $160 \mu \mathrm{m})$ bandpasses. The individual observations were calibrated, combined, and mosaicked by the SAGE team, using a custom pipeline (see Meixner et al. 2006 for further details). Because we are only interested in diffuse emission, we use point-source-subtracted versions of these images prepared by the SAGE team (see right panel of Figure 1). For the present study, we only make use of the MIPS 24,70 , and $160 \mu \mathrm{m}$ data, which have native resolutions of $\approx 5^{\prime \prime} .7$, $17^{\prime \prime}$, and $38^{\prime \prime}$, respectively. The photometric uncertainties are conservatively taken to be $5 \%, 10 \%$, and $15 \%$ at 24,70 , and $160 \mu \mathrm{m}$, respectively (see the MIPS Instrument Handbook ${ }^{7}$ ).

The majority of the emission at $24 \mu \mathrm{m}$ arises from dust heated in the vicinity of massive stars, which are the progenitors of corecollapse supernovae. Consequently, the $24 \mu \mathrm{m}$ data are used as a proxy for the CR source distribution in our phenomenological modeling. We use the full infrared data (i.e., 24, 70, and $160 \mu \mathrm{m}$ imaging) to estimate the radiation field energy density over the LMC and 30 Dor (see Section 2.4).

\subsection{Parkes + ATCA Radio Data}

We use combined $1.4 \mathrm{GHz}$ single-dish (Parkes $64 \mathrm{~m}$ ) and interferometric (ATCA) data presented in Hughes et al. (2006), where a much more detailed description of the data preparation

\footnotetext{
6 Taken from the NASA Extragalactic Database (NED; http://nedwww.ipac.caltech.edu), where there are currently 275 references for individual distance measurements to the LMC.

7 http://irsa.ipac.caltech.edu/data/SPITZER/docs/mips/ mipsinstrumenthandbook/
}

can be found. We employ a version of the radio map that has point sources removed (see left panel of Figure 1) from the map using a median filter technique (see Hughes et al. 2006) because more than $90 \%$ of such sources are expected to be background active galactic nuclei (AGNs; Marx et al. 1997). The angular resolution of the final combined mosaic is $40^{\prime \prime}$, and sets the resolution for our analysis between the radio and infrared data. At our assumed distance for the LMC, this projects to a linear scale of $\approx 10 \mathrm{pc}$.

Because we are interested in the non-thermal component of the radio data, we subtract the contribution from thermal (free-free) emission using a scaled version of the $24 \mu \mathrm{m}$ image following Equation (2) of Murphy et al. (2006b). This relation is based on the empirical correlation found between the $24 \mu \mathrm{m}$ and extinction-corrected $\mathrm{Pa} \alpha$ luminosities from star-forming regions within NGC 5194 by Calzetti et al. (2005). Although not universal (e.g., Pérez-González et al. 2006; Calzetti et al. 2007), it has proved to be a good first-order estimate of the thermal radio emission in nearby galaxies when compared to thermal fractions derived from multifrequency radio data (e.g., Murphy et al. 2008).

To check the reliability of our free-free emission estimate, we compare it with the thermal fraction derived from standard radio spectral decompositions using single-dish radio data from the literature: $1.4 \mathrm{GHz}$ (Klein et al. 1989), $2.3 \mathrm{GHz}$ (Mountfort et al. 1987), and $2.45 \mathrm{GHz}$ (Haynes et al. 1991). There is a known discrepancy between the total flux from the $1.4 \mathrm{GHz}$ single-dish map and the combined Parkes+ATCA map being used here, as Hughes et al. (2007) report a global 1.4 GHz flux density that is 1.3 times smaller than the single-dish estimate from Klein et al. (1989). This difference is largely attributed to an underestimation of the beam width for the single-dish data. Because the single-dish data were reduced and analyzed selfconsistently by Haynes et al. (1991), we assume that each map is affected in a similar way.

Using these multifrequency radio data, and assuming a constant non-thermal spectral index of $\alpha_{\mathrm{NT}} \approx 0.7$ (Haynes et al. 1991), we derive a thermal fraction at $1 \mathrm{GHz}$ that is $\approx 1.2$ times larger than the value of $25 \%$ found using the $24 \mu \mathrm{m}$ map over our region of interest (RoI) surrounding 30 Dor shown in Figure 1. This difference is consistent with results from a high-resolution radio continuum survey of M 33 (Tabatabaei et al. 2007), where a standard radio spectral decomposition was found to yield thermal fractions for $11 \mathrm{H}$ II complexes that were overestimated by an average factor of 1.5 at $8.3 \mathrm{GHz}$. However, even if we scale the $24 \mu \mathrm{m}$ derived free-free map by a factor of 1.2 to match the total thermal fraction measured by the radio spectral decomposition in our RoI, our results are not significantly affected (see Section 3.2).

\subsubsection{CR Electron Energy Estimate}

We estimate the typical energies of the CR electrons emitting the observed non-thermal $1.4 \mathrm{GHz}$ emission. For electrons propagating with a pitch angle $\theta$ in a magnetic field of strength $B$ with isotropically distributed velocities, such that $\left\langle\sin ^{2} \theta\right\rangle=2 / 3$ leading to $B_{\perp} \approx 0.82 B$, then a $\mathrm{CR}$ electron emitting at a critical frequency $v_{c}$ will have an energy

$$
\left(\frac{E_{e}}{\mathrm{GeV}}\right)=8.8\left(\frac{v_{c}}{\mathrm{GHz}}\right)^{1 / 2}\left(\frac{B}{\mu \mathrm{G}}\right)^{-1 / 2} .
$$

Integrating the free-free-corrected $1.4 \mathrm{GHz}$ flux over the region shown in Figure $1\left(S_{1.4 \mathrm{GHz}}^{\mathrm{NT}}=102.26 \mathrm{Jy}\right.$; see Section 2.4), 

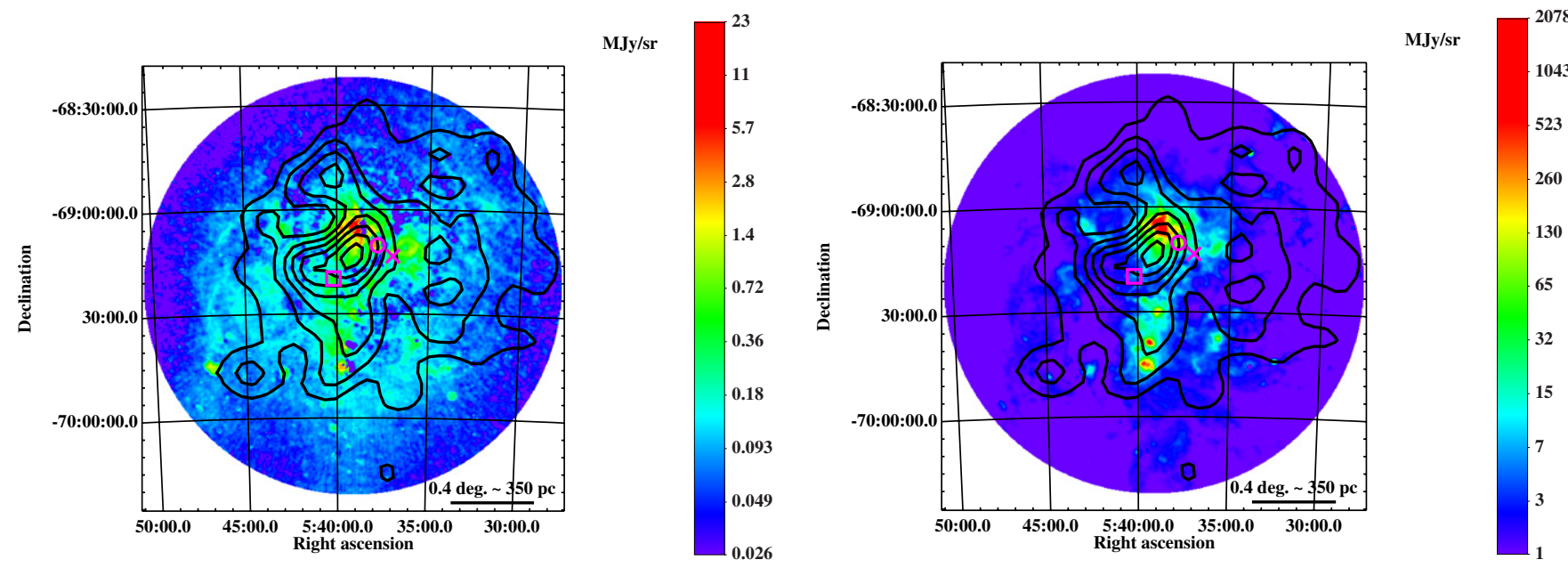

Figure 1. Point-source-subtracted $1.4 \mathrm{GHz}$ (left) and $24 \mu \mathrm{m}$ (right) maps surrounding 30 Dor with residual $1-3 \mathrm{GeV} \gamma$-ray contours starting at $0.5 \times 10^{6}$ counts sr ${ }^{-1}$ (roughly $\sim 1$ count per $0.1 \times 0.1$ pixel) with linear intervals of $0.326 \times 10^{6}$ counts sr ${ }^{-1}$ overlaid. The $1.4 \mathrm{GHz}$ map has been corrected for free-free emission. The region shown is centered at R.A. $05^{\mathrm{h}} 39^{\mathrm{m}} 07^{\mathrm{s}}$, decl. $-69^{\circ} 22^{\prime} 02^{\prime \prime}(\mathrm{J} 2000)$ and has a radius of $1^{\circ}(\approx 875 \mathrm{pc})$. The residuals between the radio/ $\gamma$-ray and infrared maps were calculated over this region (Section 2.4), as well the minimum energy magnetic field (Section 2.2.1), and the radiation field energy density (Section 2.4). In addition, we show the locations of two Crab-like pulsars, PSR J0540-6919 (square) and PSR J0537-6910 (circle), along with a known background X-ray source RX J0536.9-6913 (“×”), which are coincident with the line of sight toward 30 Dor on the $1.4 \mathrm{GHz}$ and $24 \mu \mathrm{m}$ maps.
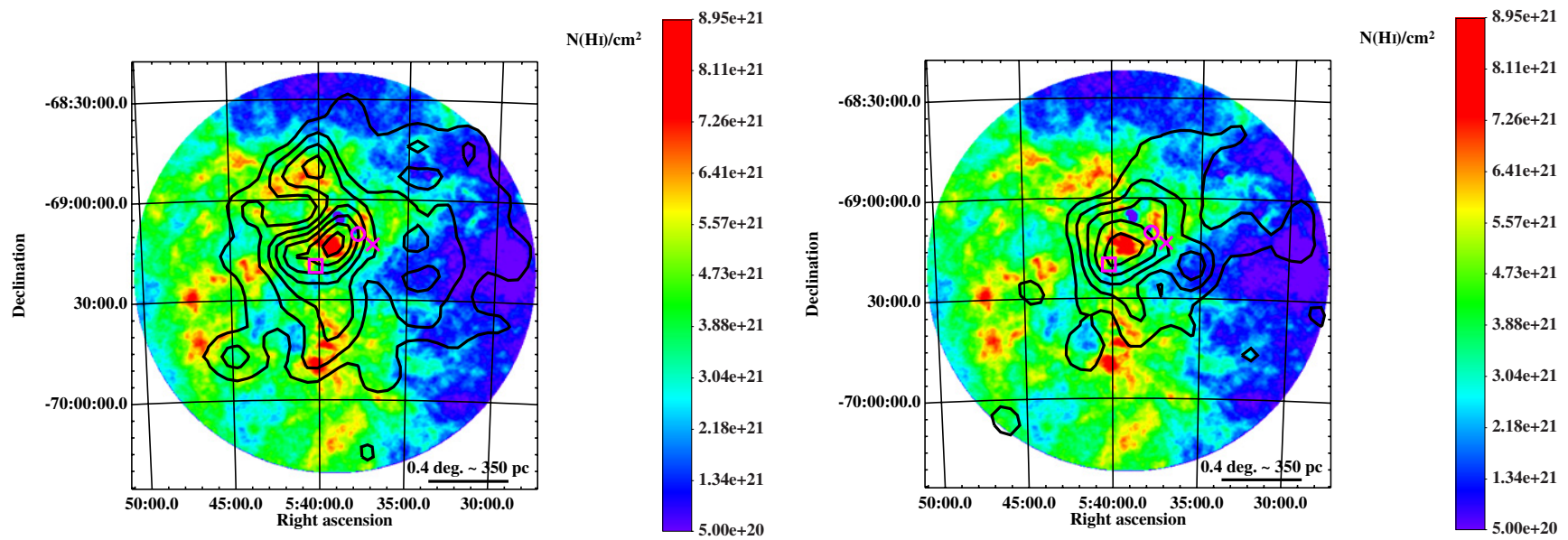

Figure 2. Same region on the sky as shown in Figure 1, but now displaying the H I column density map (Staveley-Smith et al. 2003; Kim et al. 2003). In the left panel, residual 1-3 GeV $\gamma$-ray contours are overlaid on the H I column density map with the same intervals as shown in Figure 1 . In the right panel, residual 3-10 GeV $\gamma$-ray contours are overlaid on the H I column density map starting at $0.2 \times 10^{6}$ counts sr ${ }^{-1}$ with linear intervals of $0.178 \times 10^{-6}$ counts sr $^{-1}$. Also shown are the two Crab-like pulsars, PSR J0540-6919 (square) and PSR J0537-6910 (circle), along with a known background X-ray source RX J0536.9-6913 (“×”), which are coincident with the line of sight toward 30 Dor.

we use the revised minimum energy calculation of Beck \& Krause (2005), assuming a proton-to-electron number density ratio of $K_{0} \approx 100 \pm 50$, a non-thermal spectral index of $\alpha_{\mathrm{NT}} \approx$ $0.7 \pm 0.1$, and a path length of $l \approx 1 \pm 0.5 \mathrm{kpc}$, to calculate a minimum energy magnetic field strength of $\sim 11 \pm 4 \mu \mathrm{G}$. Using this range of values in Equation (1), our 1.4 GHz maps are most sensitive to $\sim 3 \pm 0.5 \mathrm{GeV} \mathrm{CR}$ electrons. If the minimum energy condition does not hold, and we instead assume an extreme range in $B$, such as 3-50 $\mu \mathrm{G}$, the corresponding CR electron energies will range from 6 to $1.5 \mathrm{GeV}$. However, the (in)validity of the minimum energy assumption is uncertain and often assumed (as we do in this paper). We briefly discuss the impact of this on the derived properties of the diffusion coefficient in Section 4.2.1.

\subsection{Fermi-LAT Data}

The Fermi-LAT instrument, event reconstruction, and response are described in Atwood et al. (2009). In this paper, we use events and instrument response functions (IRFs) for the standard low-background "Clean" events corresponding to the Pass 7 event selections. ${ }^{8}$ To minimize the contribution from the very bright Earth limb, we restrict the event selection and exposure calculation to zenith angles $<100^{\circ}$. We selected all front-converting events, for which the point-spread function (PSF) is narrowest, within a $20^{\circ}$ square RoI centered on R.A. $05^{\mathrm{h}} 38^{\mathrm{m}} 42^{\mathrm{s}}$, decl. $-69^{\circ} 06^{\prime} 03^{\prime \prime}$ (J2000) for $\approx 32$ months of sky survey data from 2008 August 4 until 2011 March 24. Exposure maps and the PSF for the pointing history of the observations were generated using the standard Fermi-LAT ScienceTools package available from the Fermi Science Support Center. ${ }^{9}$ The exposure of the instrument over the RoI for the data taking period used in this analysis is very uniform.

We constructed foreground-subtracted $\gamma$-ray maps over the same region using the standard Fermi-LAT diffuse

\footnotetext{
8 We use the P7V6_CLEAN event class in this work; see http://fermi.gsfc.nasa.gov/ssc/data/analysis/documentation/Pass7_usage.html. 9 http://fermi.gsfc.nasa.gov/ssc/data/analysis/
} 
emission model ${ }^{10}$ together with the appropriate isotropic background model and $\gamma$-ray point sources from the Fermi-LAT Second Source Catalog (Nolan et al. 2012), forward folding the combination with the exposure and PSF to obtain the total counts expected for these foregrounds. These were subtracted from the data and the residual emission for the $1-3 \mathrm{GeV}$ energy interval is shown in Figure 1 overlaid on the free-free-corrected $1.4 \mathrm{GHz}$ and $24 \mu \mathrm{m}$ maps. The effective $68 \%$ containment radius for the Fermi-LAT PSF over this energy bin for the event selection used is $\approx 0.50(\approx 440 \mathrm{pc})$, assuming an $E_{\gamma}^{-2.1}$ spectrum for the $\gamma$-ray emission, which is a sufficient approximation to the spectral shape determined by Abdo et al. (2010b) for the LMC $\gamma$-ray emission over this energy range. ${ }^{11}$ Note that the effective FWHM of the Fermi-LAT PSF over this energy bin is $\approx 0.38(\approx 330 \mathrm{pc})$, significantly smaller than the $68 \%$ containment radius quoted above.

Similarly, we constructed a map for the 3-10 GeV residual emission. This is shown in Figure 2 where we overlay the 1-3 and 3-10 GeV contours on the Hi column density data (Staveley-Smith et al. 2003; Kim et al. 2003). The effective $68 \%$ containment radius for this map is $\approx 0.24(\approx 210 \mathrm{pc})$, with an effective FWHM of $\approx 0.19(\approx 170 \mathrm{pc})$, again assuming an $E_{\gamma}^{-2.1}$ spectrum for the $\gamma$-ray emission over this energy bin. However, the 3-10 GeV map has much lower statistics, even near 30 Dor, contrasting with the $1-3 \mathrm{GeV} \gamma$-ray map, which has $\gtrsim 3$ times more events. Therefore, we base the majority of our spatial analysis on the $1-3 \mathrm{GeV}$ map, and only use the higher energy $\gamma$-ray data for testing the energy dependence of the CR propagation (see Section 4.2.2).

\subsubsection{CR Nuclei Energy Estimate}

As for electrons and the $1.4 \mathrm{GHz}$ emission, we estimate the typical energies of the CR nuclei emitting $\gamma$ rays for the energy ranges that we consider in this paper. For non-AGN-dominated star-forming galaxies like the Milky Way and the LMC, the assumption that the $\mathrm{CR}$ nuclei are the predominant relativistic particle population producing the $\gamma$-ray emission in the FermiLAT energy range is reasonable (Strong et al. 2010).

We define the function

$$
\begin{aligned}
G\left(E_{\gamma}, E_{p}\right)= & \left(\int_{E_{p}}^{\infty} d E_{p}^{\prime} \beta \frac{d J_{p}}{d E_{p}^{\prime}} \frac{d \sigma_{p p}\left(E_{\gamma}, E_{p}^{\prime}\right)}{d E_{\gamma}}\right) \\
& /\left(\int_{E_{p}^{\min }\left(E_{\gamma}\right)}^{\infty} d E_{p}^{\prime} \beta \frac{d J_{p}}{d E_{p}^{\prime}} \frac{d \sigma_{p p}\left(E_{\gamma}, E_{p}^{\prime}\right)}{d E_{\gamma}}\right)-\frac{1}{2},
\end{aligned}
$$

where $E_{p}$ and $E_{\gamma}$ are the proton and $\gamma$-ray energies, $\beta=v / c$, $d J_{p} / d E_{p}^{\prime} \propto E_{p}^{\prime-\alpha}$ is the CR proton flux, $d \sigma_{p p} / d E_{\gamma}$ is the differential cross section for $\gamma$-ray production calculated as in Moskalenko \& Strong (1998), and $E_{p}^{\mathrm{min}}$ is the minimum proton energy required to produce a photon of energy $E_{\gamma}$. The effective proton energy, $E_{p}^{\text {eff }}$, for a given $E_{\gamma}$ is given by the root of $G\left(E_{\gamma}, E_{p}\right)$ so that contributions to the $\gamma$-ray flux at $E_{\gamma}$ below and above $E_{p}^{\text {eff }}$ are equal. The effective proton energy is a function of the proton spectral index $E_{p}^{\text {eff }}=E_{p}^{\text {eff }}(\alpha)$, which we show for different $\alpha$ for the $1-3 \mathrm{GeV}$ and $3-10 \mathrm{GeV}$ energy ranges in Table 1 . We also show the effective (average) $\gamma$-ray

\footnotetext{
${ }^{10}$ http://fermi.gsfc.nasa.gov/ssc/data/access/lat/BackgroundModels.html 11 Weighting with an $E_{\gamma}^{-2.8}$ spectrum gives a marginally different result, being larger by $\approx 3.5 \%$.
}

Table 1

Effective $\gamma$-ray and Proton Energies for Different $\gamma$-ray Energy Ranges

\begin{tabular}{lcccc}
\hline \hline \multirow{2}{*}{\begin{tabular}{l} 
Index \\
\cline { 2 - 5 }
\end{tabular}} & \multicolumn{4}{c}{$\gamma$-ray Energy Range } \\
\cline { 2 - 5 } & $\begin{array}{c}E_{\gamma}^{\text {eff }} \\
(\mathrm{GeV})\end{array}$ & $\begin{array}{c}E_{p}^{\text {eff }} \\
(\mathrm{GeV})\end{array}$ & $\begin{array}{c}E_{\gamma}^{\text {eff }} \\
(\mathrm{GeV})\end{array}$ & $\begin{array}{c}E_{p}^{\text {eff }} \\
(\mathrm{GeV})\end{array}$ \\
\hline 2.1 & 1.63 & 22.4 & 5.10 & 69.2 \\
2.2 & 1.61 & 19.5 & 5.04 & 60.4 \\
2.3 & 1.60 & 17.2 & 4.99 & 53.6 \\
2.4 & 1.58 & 15.4 & 4.93 & 48.1 \\
2.5 & 1.60 & 14.1 & 4.87 & 43.6 \\
2.6 & 1.55 & 12.5 & 4.82 & 39.8 \\
2.7 & 1.54 & 11.5 & 4.77 & 36.6 \\
2.8 & 1.53 & 10.5 & 4.72 & 33.9 \\
\hline
\end{tabular}

energy for the 1-3 GeV and 3-10 GeV energy ranges, calculated assuming the same spectral index for the $\gamma$ rays as for the protons. This assumption is valid for thin-target interactions by the CR nuclei with gas, which applies for the ISM surrounding 30 Dor and the greater LMC.

The effective proton energy changes by a factor of $\sim 2$ when the power-law index of the proton spectrum changes from 2.1 to 2.8 , while the effective energy of $\gamma$ rays does not change appreciably. For the $\gamma$-ray energies, this is not surprising because the effective energy is calculated over a relatively narrow energy bin. The effective proton energy change is a combination of the fairly steep rise in the inclusive pion production cross section as the momentum of the interacting proton increases, approximately $\propto E^{0.5}$ between 10 and $100 \mathrm{GeV}$ (see, e.g., Figure 2(a) from Dermer 1986). When the proton spectrum is steep (e.g., with an index $\sim 2.8$ ), this rise is not large enough to compensate for the steeply falling number of high-energy protons. When the proton spectrum is flat (e.g., with an index 2.1) the rise in the inclusive cross section increases the contribution of high-energy protons, making the $E_{p}^{\mathrm{eff}}$ significantly larger.

\subsection{Image Registration and Smearing Analysis}

All images were cropped to a common field-of-view and regridded to a common pixel scale. To properly compare each image at the same resolution, maps were convolved with an appropriate PSF. For the radio and infrared $(24,70$, and $160 \mu \mathrm{m})$ data, we convolve all images to the resolution of the $1.4 \mathrm{GHz}$ image, which has an FWHM of 40", using a Gaussian beam having an FWHM equal to the quadrature difference between the final and original FWHM values of each image.

To match the resolution of the $24 \mu \mathrm{m}$ data to that of the $\gamma$-ray maps, we convolve the $24 \mu \mathrm{m}$ image (at its native resolution) with the in-flight Fermi-LAT PSF, described in Section 2.3. Because the FWHM of the $24 \mu \mathrm{m}$ PSF is $<1 \%$ of the effective FWHM over the $\gamma$-ray energy bins used in our analysis, we do not attempt to correct for this additional broadening.

Next, we apply our image smearing analysis (described below) to the resolution-matched images to determine the extension of the non-thermal radio and $\gamma$-ray morphologies relative to the $24 \mu \mathrm{m}$ morphology. The procedure largely follows that described in Murphy et al. (2006b, 2008), where we refer the reader for a more detailed description. Our underlying assumption is that the only difference between these distributions is due to the diffusion and energy losses of the CRs because of the common origin for the CR leptons and nuclei. We focus on the $24 \mu \mathrm{m}$ data in the smearing analysis rather than the $70 \mu \mathrm{m}$ data, as was done by, e.g., Murphy et al. (2008), because it traces warmer 
dust and has been found to be more peaked near star-forming regions than the $70 \mu \mathrm{m}$ morphology (Helou et al. 2004; Murphy et al. 2006a). For instance, typical $24 \mu \mathrm{m}$ knots surrounding 30 Dor tend to be $\sim 10 \mathrm{pc}$ in size. Thus, the $24 \mu \mathrm{m}$ maps likely act as a better source function for recently accelerated CRs.

To summarize the procedure, we convolve the entire $24 \mu \mathrm{m}$ image of the LMC by a parameterized kernel $\kappa(\mathbf{r})$ and compute the residuals between the free-free-corrected $1.4 \mathrm{GHz}$ and smoothed $24 \mu \mathrm{m}$ maps. The smoothing kernel is a function of a two-dimensional position vector $\boldsymbol{r}$, having a magnitude of $r=\left(x^{2}+y^{2}\right)^{1 / 2}$, where $x$ and $y$ are the right ascension and declination offsets on the sky, respectively. We investigate both exponential and Gaussian kernels because a preference of kernel type may suggest different CR transport effects. The exponential and Gaussian kernels have the forms of $\kappa(\boldsymbol{r})=e^{-\boldsymbol{r} / l}$ and $\kappa(\boldsymbol{r})=e^{-\boldsymbol{r}^{2} / l^{2}}$, respectively, where $l$ is the $e$-folding scale length. Thus, for the Gaussian kernel, $\sigma^{2}=l^{2} / 2$.

Gaussian kernels suggest a simple random walk diffusion scenario. On the other hand, exponential kernels can arise as the result of energy losses or escape on timescales comparable to diffusion, modifying the Gaussian kernels (e.g. Bicay \& Helou 1990; Helou \& Bicay 1993). Because the LMC lacks a welldefined disk, and has a low inclination $\left(i \approx 31^{\circ}\right.$; Nikolaev et al. 2004), we assume spherical symmetry for 30 Dor. Hence, there is no need to modify the position vector by geometric factors.

We calculate the normalized-squared residuals between the radio and the infrared images

$$
\phi(l)=\frac{\Sigma\left[Q^{-1} \tilde{I}_{j}(l)-R_{j}\right]^{2}}{\Sigma R_{j}^{2}},
$$

where $R$ is the radio image with free-free emission removed, $\tilde{I}(l)$ is the infrared image smoothed by a kernel with scale length $l, Q=\Sigma I_{j} / \Sigma R_{j}$ is used as a normalization factor (i.e., the global $24 \mu \mathrm{m} / 1.4 \mathrm{GHz}$ ratio), and the subscript $j$ indexes each pixel. Only pixels detected with a significance of $>3 \sigma$ of the rms noise in each map are used in the calculation of the residuals. The minimum in $\phi$ defines the best-fit scale length, which is taken to be the typical distance traveled by the CR electrons. Similarly, we perform the same procedure using the $24 \mu \mathrm{m}$ and 1-3 GeV $\gamma$-ray maps to determine the typical distance traveled by the CR nuclei.

For this procedure, generically, if there is no broadening due to propagation effects, the minimum of Equation (3) will be at $l=0$, because both the radio and $\gamma$-ray data have already been reduced to the same angular resolution. Below, we obtain kernel scale lengths indicating that significant smoothing of the $24 \mu \mathrm{m}$ image is required to improve the match with the radio and $\gamma$-ray images. While the scale lengths obtained for the radio $/ 24 \mu \mathrm{m}$ residual analysis are much larger than the angular resolution of these maps, for the $\gamma$-ray $/ 24 \mu \mathrm{m}$ data, the derived scale lengths are comparable to the angular resolution after convolution with the Fermi-LAT PSF. The technique is capable of detecting scale lengths that are a small fraction of the resolution (i.e., $\ll \Delta l=50 \mathrm{pc}$, which is the scale length step size used in the analysis), and is more sensitive for higher signal-to-noise ratio maps. We emphasize that the kernel scale lengths obtained in this case are in addition to the smoothing of the $24 \mu \mathrm{m}$ data to match the Fermi-LAT PSF. If there was no additional effect from propagation, the derived scale lengths would be zero, whereas we detect a meaningfully non-zero scale length.

The uncertainty in $\phi$ is estimated by numerically propagating the uncertainties in the input images as measured by the $1 \sigma \mathrm{rms}$ noise of each map, with the uncertainty for the best-fit scale length then estimated as the range in scale length corresponding to that from $\min (\phi)$ to $\min (\phi)+\operatorname{unc}(\phi)$ along the residual curve. We note that this places a lower limit on the uncertainty for the best-fit scale lengths since there is additional uncertainty on the $1.4 \mathrm{GHz}$ thermal fraction estimation and foreground subtraction for the $\gamma$-ray maps. The calculation of the residuals and photometry was carried out within an aperture having a radius of $1^{\circ}(\approx 875 \mathrm{pc})$, encompassing 30 Dor, centered at $05^{\mathrm{h}} 39^{\mathrm{m}} 07^{\mathrm{s}},-69^{\circ} 22^{\prime} 02^{\prime \prime}$ (J2000, see Figure 1).

We use the 24,70 , and $160 \mu \mathrm{m}$ photometry to calculate the total infrared (IR, 8-1000 $\mu \mathrm{m}$ ) luminosity over this region by fitting these data to the spectral energy distribution (SED) models of Dale \& Helou (2002) and integrating the best-fit SED between 8 and $1000 \mu \mathrm{m}$. The individual 24, 70, and $160 \mu \mathrm{m}$ flux densities are $0.42 \pm 0.02,4.1 \pm 0.41$, and $8.0 \pm 1.7 \times 10^{4} \mathrm{Jy}$, respectively. In addition to photometric uncertainties, the above errors include a term for the mean rms noise values in the convolved 24, 70, and $160 \mu \mathrm{m}$ maps, which are measured to be $\sim 0.045,0.685$, and $13.2 \mathrm{MJy} \mathrm{sr}^{-1}$, respectively. We obtain an IR luminosity of $L_{\mathrm{IR}}=1.15 \pm 0.12 \times 10^{42} \mathrm{erg} \mathrm{s}^{-1}$ $\left(3.00 \pm 0.31 \times 10^{8} L_{\odot}\right)$. Taking this value, we estimate the corresponding radiation field energy density

$$
U_{\mathrm{rad}} \approx \frac{2 \pi}{c} I_{\mathrm{bol}} \gtrsim \frac{L_{\mathrm{IR}}}{2 A_{\mathrm{IR}} c}\left(1+\sqrt{\frac{3.8 \times 10^{42}}{L_{\mathrm{IR}}}}\right),
$$

where $I_{\text {bol }}$ is the bolometric surface brightness, $c$ is the speed of light, and $A_{\mathrm{IR}} \approx 2.4 \mathrm{kpc}^{2}$ is the area over which the photometry was measured. All quantities in Equation (4) are in cgs units. This calculation is for radiation emitted near the surface of a semitransparent body, and the parenthetical term provides an empirically derived correction for non-absorbed UV emission (Bell 2003), resulting in a value of $U_{\mathrm{rad}} \approx$ $2.39 \pm 0.17 \times 10^{-12} \mathrm{erg} \mathrm{cm}^{-3}\left(1.49 \pm 0.11 \mathrm{eV} \mathrm{cm}^{-3}\right)$ averaged over the volume considered.

\section{RESULTS}

We present our results from comparing the morphologies of 30 Dor as measured by warm dust emission at $24 \mu \mathrm{m}$, synchrotron emission at $1.4 \mathrm{GHz}$, and $\gamma$-ray emission at 1-3 GeV. We compare the morphologies both qualitatively, through a visual inspection, and quantitatively using the image smearing analysis described above.

\section{1. $24 \mu \mathrm{m}, 1.4 \mathrm{GHz}$, and 1-3 GeV $\gamma$-Ray Morphologies}

Figure 1 shows the residual $1-3 \mathrm{GeV} \gamma$-ray emission overplotted as contours on the free-free-corrected $1.4 \mathrm{GHz}$ radio and $24 \mu \mathrm{m}$ warm dust emission in the left and right panels, respectively. While there is a general correspondence between the $\gamma$-ray, radio, and infrared emission, the peak of the $\gamma$-ray emission is clearly offset from the peak of the radio and infrared emission, which appear to be nearly co-spatial. This lack of correspondence between the peak of the $\gamma$-ray emission with the radio and infrared emission leads one to question whether the observed $\gamma$-ray emission is indeed associated with 30 Dor.

In Abdo et al. (2010b), it was noted that $\gamma$-ray emission near 30 Dor may have a non-negligible contribution from two Crab-like pulsars, PSR J0540-6919 $\left(05^{\mathrm{h}} 40^{\mathrm{m}} 11^{\mathrm{s}} .2,-69^{\circ} 19^{\prime} 54^{\prime \prime}\right.$; $\mathrm{J} 2000)$ and PSR J0537-6910 $\left(05^{\mathrm{h}} 37^{\mathrm{m}} 47^{\mathrm{s}} .4,-69^{\circ} 10^{\prime} 20^{\prime \prime}\right.$; $\mathrm{J} 2000$ ). In addition, there is a background X-ray source 


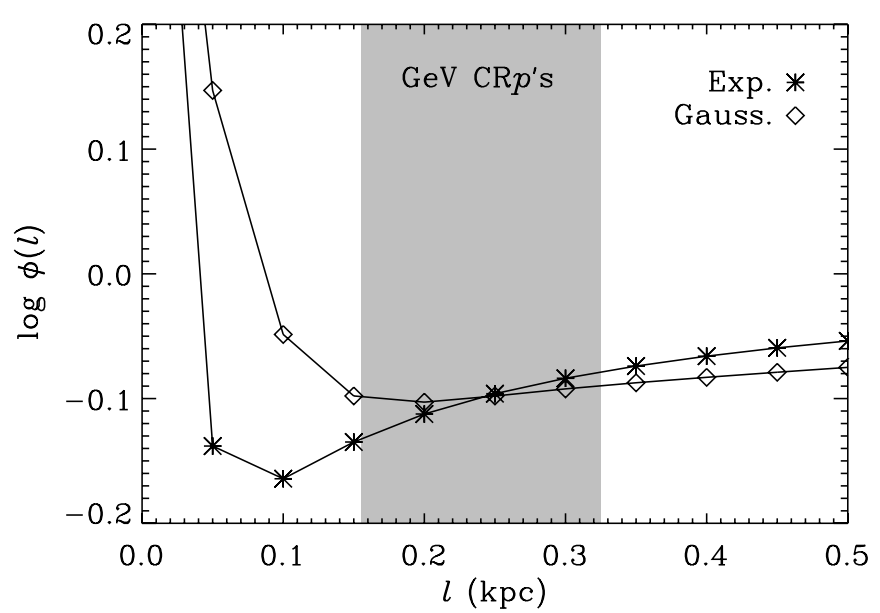

Figure 3. Residuals between the free-free-corrected $1.4 \mathrm{GHz}$ and smoothed $24 \mu \mathrm{m}$ (as defined in Section 2.4) plotted as a function of both exponential (asterisks) and Gaussian (diamonds) of kernel scale lengths. The shaded region indicates the extrapolated propagation length for $\mathrm{GeV}$ protons based on the compact morphology of the $\gamma$-ray emission observed by the Fermi-LAT (Abdo et al. 2010b). The value reported by Abdo et al. (2010b) is the $\sigma$ from a modeled Gaussian. The values for the Gaussian scale lengths agree within errors.

(RX J0536.9-6913: $05^{\mathrm{h}} 36^{\mathrm{m}} 57^{\mathrm{s}} .8,-69^{\circ} 13^{\prime} 26^{\prime \prime}$; J2000) that is also coincident with the line of sight toward 30 Dor, which could also contribute to the observed emission. The locations of these point sources are shown in Figures 1 and 2, and are near, but not at, the peak of the $1-3$ and $3-10 \mathrm{GeV} \gamma$-ray emission. It is also interesting to see that the 1-3 and 3-10 $\gamma$-ray contours appear to peak close to a region of high $\mathrm{H}$ I column density in Figure 2. It is possible to subtract fitted $\gamma$-ray point sources at the locations of the pulsars and AGN, resulting in a situation where 30 Dor does not exhibit $\gamma$-ray emission in excess of the subtracted foreground model. The implications of such a scenario are described in Section 4.1. However, we note that there is no detection of pulsed $\gamma$-ray emission so far reported from either of the pulsars. Also, no variability is detected from the region during our period of analysis. If RX J0536.9-6913 is a $\gamma$-ray-emitting AGN, the lack of variability makes it difficult to determine if this candidate source contributes significantly in our RoI.

\subsection{Results from the Smearing Analysis}

In Figure 3, we plot the residuals ( $\phi$, see Section 2.4) between the free-free-corrected $1.4 \mathrm{GHz}$ and smoothed $24 \mu \mathrm{m}$ maps as a function of exponential and Gaussian kernel scale lengths. The residuals between the free-free-corrected $1.4 \mathrm{GHz}$ and $24 \mu \mathrm{m}$ maps are decreased by more than a factor of $\sim 2$ after smoothing the $24 \mu \mathrm{m}$ map using either exponential or Gaussian kernels. A slightly larger improvement is found by using an exponential kernel, suggesting energy losses and/or escape may be important for the CR electrons on timescales comparable to the diffusion timescale. The corresponding bestfit exponential and Gaussian kernel scale lengths are $100_{-10}^{+10}$ and $200_{-55}^{+100} \mathrm{pc}$, respectively. We note that the estimated $\sim 3 \mathrm{GeV}$ $\mathrm{CR}$ electron propagation length reported here assuming random walk diffusion (i.e., using a Gaussian kernel) is consistent within errors to the $\mathrm{GeV} \mathrm{CR}$ proton confinement length reported by Abdo et al. (2010b), whose estimate assumed Gaussian profiles (see Figure 3). Abdo et al. (2010b) report the Gaussian $\sigma$ to be $\sigma=170 \pm 60 \mathrm{pc}$, so we multiply this number by $\sqrt{2}$ for proper

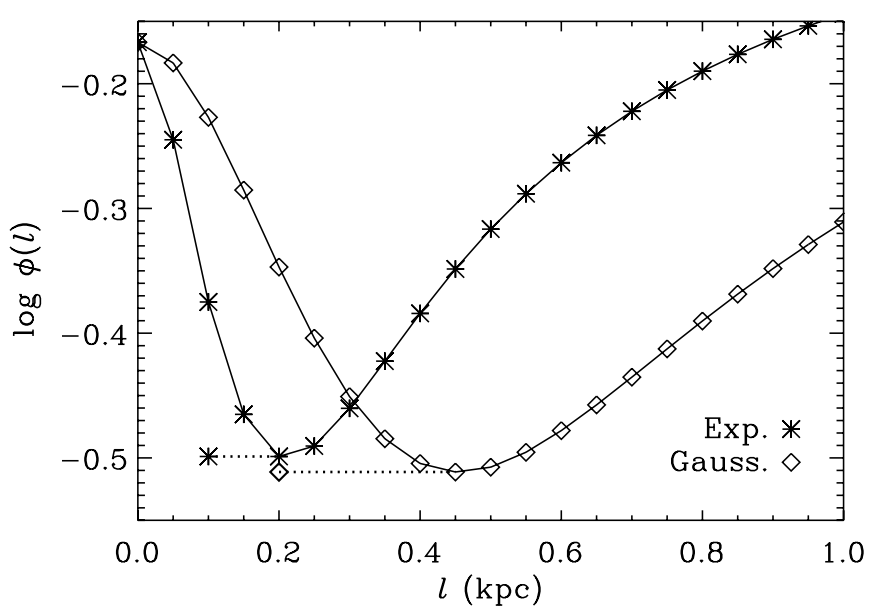

Figure 4. Residuals between the 1-3 GeV $\gamma$-ray and smoothed $24 \mu \mathrm{m}$ images (as defined in Section 2.4) plotted as a function of both exponential (asterisks) and Gaussian (diamonds) of kernel scale lengths. The asterisk and diamond attached to the horizontal dotted lines indicate the location of the best-fit scale length measured for the CR electrons using exponential and Gaussian kernels (see Figure 3).

comparison with the scale length definition for our Gaussian kernel.

As discussed in Section 2.2, we test how our results are affected by assuming that the thermal fraction of the $1.4 \mathrm{GHz}$ radio map is being underestimated by the $24 \mu \mathrm{m}$ maps relative to what is derived using single-dish radio data at $1.4,2.3$, and $2.45 \mathrm{GHz}$. By scaling the $24 \mu \mathrm{m}$ derived free-free maps by a factor of 1.2 to match the total thermal fraction measured by the radio spectral decomposition, and repeating the smearing analysis, we find that the best-fit exponential scale length is still $100 \mathrm{pc}$, while the best-fit Gaussian scale length is slightly increased to $250 \mathrm{pc}$. A larger improvement is again found using an exponential kernel relative to a Gaussian kernel. The results therefore do not appear to be significantly affected by increasing the thermal fraction estimate to match that from the radio spectral decomposition method.

Similarly, in Figure 4, we plot the residuals between the 1-3 GeV $\gamma$-ray and smoothed $24 \mu \mathrm{m}$ maps as a function of exponential and Gaussian kernel scale lengths. The residuals between the $1-3 \mathrm{GeV}$ and $24 \mu \mathrm{m}$ maps are decreased by a factor of $\sim 2$ after smoothing the $24 \mu \mathrm{m}$ map using either exponential or Gaussian kernels. While a slightly larger improvement is found by using exponential kernels in the comparison between the $1.4 \mathrm{GHz}$ and $24 \mu \mathrm{m}$ maps, we do not find a preference in kernel type for the $1-3 \mathrm{GeV} \gamma$-ray map. The corresponding bestfit exponential and Gaussian kernel scale lengths are $200_{-10}^{+40}$ and $450_{-50}^{+65} \mathrm{pc}$, respectively. These best-fit scale lengths are significantly larger (i.e., a factor of $\sim 2$ ) than what was measured for the $1.4 \mathrm{GHz}$ maps. Because the $1.4 \mathrm{GHz}$ and $1-3 \mathrm{GeV}$ $\gamma$-ray maps probe different energy CR particle populations, the corresponding differences in their best-fit scale lengths may arise from different diffusion speeds. This scenario is discussed in Section 4.2.

\section{DISCUSSION}

Using a phenomenological image smearing model, we have estimated the typical propagation lengths of $\sim 3 \mathrm{GeV} \mathrm{CR}$ electrons and $\sim 20 \mathrm{GeV} \mathrm{CR}$ nuclei by comparing the spatial distributions of smoothed $24 \mu \mathrm{m}$ maps images with (free-free- 
corrected) $1.4 \mathrm{GHz}$ and (foreground-subtracted) $1-3 \mathrm{GeV} \gamma$-ray maps, respectively. From the analysis, there is a difference between the typical distances traveled by $\sim 3 \mathrm{GeV}$ CR electrons and $\sim 20 \mathrm{GeV}$ protons (assuming a proton energy index of 2.1), where the $20 \mathrm{GeV} \mathrm{CR}$ protons are found to travel $\sim 2$ times further, on average, independent of kernel type. However, it is additionally worth including a brief discussion of the physical implications for the case where 30 Dor may not be emitting in $\gamma$ rays.

\subsection{The Case for a Dark 30 Dor in $\gamma$-Rays}

As stated in Section 3.1, there are both pulsars and a background X-ray-emitting source that may contribute to the observed $\gamma$-ray emission toward 30 Dor. For this case, the situation has 30 Dor emitting at $1.4 \mathrm{GHz}$, therefore containing diffusing CR electrons, but no observational signature for the $\mathrm{CR}$ nuclei. Explaining this would require the $\mathrm{CR}$ nuclei to escape the system without interacting with the interstellar gas.

Taking the above estimate for the bolometric luminosity from 30 Dor (i.e., the IR luminosity given in Section 2.4 corrected by the parenthetical term in Equation (4) for non-absorbed UV emission) together with the updated SFR calibrations given in Murphy et al. (2011), we estimate a corresponding SFR of $\sim 0.15 M_{\odot} \mathrm{yr}^{-1}$. This value is consistent with others in the literature (e.g., Hughes et al. 2007; Harris \& Zaritsky 2009; Lawton et al. 2010). Assuming this value and the sensitivity of the Fermi-LAT data used in our analysis, the absence of $\gamma$-ray emission from 30 Dor clearly disagrees with the empirical correlations between SFR or the product of supernova rate and the gas mass with $\gamma$-ray luminosity found for the local group and nearby starbursts (e.g., Abdo et al. 2010a; Lenain $\&$ Walter 2011). Furthermore, theoretical expectations of such scaling relations and their implications for the contribution by normal star-forming galaxies to the diffuse $\gamma$-ray extragalactic background (e.g., Pavlidou \& Fields 2001; Fields et al. 2010) would then be questionable. However, because there is currently no strong evidence linking the $\gamma$-ray emission from 30 Dor to the known point sources in the field, we discuss the physical interpretation of our analysis with the assumption that it arises from 30 Dor.

\subsection{Cosmic-ray Propagation from 30 Dor}

To interpret our findings on the kernel scale lengths, we must consider the dependence of gas density on the synchrotron and $\gamma$-ray emission. The diffuse $\gamma$ rays in the energy range that we have used (1-3 GeV) are predominantly from CR nuclei interacting with the interstellar gas, and therefore $S_{\gamma} \propto n_{p} n_{\mathrm{ISM}}$ where $n_{p}$ and $n_{\mathrm{ISM}}$ are the densities of the CR nuclei and gas, respectively. The diffuse synchrotron emission is proportional to the CR electron density, $n_{e}$, and the square of the magnetic field strength, $B$, i.e., $S_{\text {sync }} \propto n_{e} B^{2}$. Assuming flux-freezing scaling of $B \propto \sqrt{n_{\text {ISM }}}$ (e.g., Ruzmaikin et al. 1988; Niklas \& Beck 1997; Crutcher 1999), the synchrotron emission becomes proportional to the product of the electron density and the density of the interstellar gas, $S_{\text {sync }} \propto n_{e} n_{\text {ISM. This simple scaling argument }}$ therefore suggests that the differences in appearance of the $\gamma$-ray and radio images are due to differences in the distributions of the CR nuclei and electrons. This was tested by normalizing the $\gamma$-ray and free-free-corrected radio images by the $\mathrm{HI}_{\mathrm{I}}$ column density map before applying the smearing analysis, recovering results consistent with those described in Section 3.2.

\subsubsection{Cosmic-ray Transport Properties}

We assume that the transport of the CRs can be described by a simple random walk process with a rigidity-dependent spatial diffusion coefficient $D_{R}$ (e.g., Ginzburg et al. 1980). The diffusion coefficient is defined as

$$
D_{R}=D_{0}\left(\frac{R}{\mathrm{GV}}\right)^{\delta} \approx \frac{l_{\mathrm{diff}}^{2}}{\tau_{\mathrm{diff}}}
$$

where $D_{0}$ is the normalization constant and $l_{\text {diff }}$ is the characteristic distance that CRs travel after a time $\tau_{\text {diff }}$. For our Gaussian kernels, we can relate $l_{\text {diff }}$ to the corresponding best-fit scale lengths such that $l_{\text {diff }}^{2}=\sigma^{2}=l^{2} / 2$.

If we assume that the CR electrons and nuclei are injected by the same source(s) and have been propagating through the ISM for the same length of time, we find that the $\sim 20 \mathrm{GeV}$ $\mathrm{CR}$ nuclei diffusion coefficient is $\sim 4$ times larger than that for the $\sim 3 \mathrm{GeV}$ CR electrons. Solving Equation (6) for $\delta$, we find that the diffusion coefficient scales as $(R / \mathrm{GV})^{0.69 \pm 0.15}$ from the exponential best-fit scale lengths and as $(R / \mathrm{GV})^{0.81 \pm 0.30}$ using the Gaussian best-fit scale lengths. Errors on $\delta$ were estimated by a standard Monte Carlo approach using the uncertainties in the best-fit scale lengths. ${ }^{12}$ If the minimum energy assumption does not hold, and we instead consider the extreme range of $B \approx 3-50 \mu \mathrm{G}$ described earlier in Section 2.2.1, we find corresponding $\delta$ values of $\approx 0.5-1.2$.

These model-independent values of $\delta$ are consistent with the value of $\sim 0.6-0.7$ used in empirical diffusion models to fit the observed secondary-to-primary ratios, typically boron-tocarbon $(\mathrm{B} / \mathrm{C})$, for the Milky Way. In addition, our derived values for $\delta$ generally exceed those for physically motivated turbulence theories, being larger than the nominal value of $1 / 3$ for Kolomogorov (Kolmogorov 1941), and marginally consistent with the value of $1 / 2$ for Iroshnikov-Kraichnan (Iroshnikov 1964; Kraichnan 1965) turbulence spectra. However, the values for the Milky Way are found for a large volume in a spiral galaxy, while the results of our analysis are for a single, highly active star-forming region: 30 Dor exhibits a complex network of kinematic features including slow $\left(v \lesssim 100 \mathrm{~km} \mathrm{~s}^{-1}\right.$ ) and fast $\left(100 \mathrm{~km} \mathrm{~s}^{-1} \lesssim v \lesssim 300 \mathrm{~km} \mathrm{~s}^{-1}\right)$ expanding shells powered by stellar winds from young massive stars and supernovae (Chu \& Kennicutt 1994).

Because of strong radiative energy losses as they propagate, the corresponding diffusion lengths for the $\sim 3 \mathrm{GeV}$ CR electrons may be underestimated by our best-fit scale lengths, which would result in an overestimate for $\delta$. This is suggested by the fact that an exponential kernel resulted in a lower minimum residual between the $1.4 \mathrm{GHz}$ and $24 \mu \mathrm{m}$ maps than a Gaussian kernel. However, as discussed below, the additional cooling of electrons may not significantly affect our estimate for $\delta$.

Taking the average derived value for $\delta$ (i.e., 0.75), together with the age of the current star formation activity responsible for supernova remnants accelerating the CRs, we can estimate the diffusion coefficient normalization factor $D_{0}$. The 30 Dor complex is known to contain many non-coeval stellar populations ranging in age from $<1$ to 10 Myr from Hubble Space Telescope spectroscopy (Walborn \& Blades 1997). Recently, using a Bayesian analysis, Martínez-Galarza et al. (2011) fit Spitzer Infrared Spectrograph data for 30 Dor using mid-infrared SED

\footnotetext{
$12 \delta$ was calculated by taking 1000 random samples, picking best-fit scale lengths from a normal distribution having a dispersion set by their lower- and upper-bound uncertainties. The standard deviation of this set is taken as the uncertainty on $\delta$.
} 
models (continuum + lines), arriving at a luminosity-weighted age for the system of $\sim 3$ Myr. This suggests that CRs associated with this star-forming event have been accelerated in the supernova remnants of very massive O-stars, and that the bulk of CRs have yet to be accelerated by the supernova remnants from the more numerous and less massive (i.e., $\sim 8 M_{\odot}$ ) stars with lifetimes of $\sim 30$ Myr. Note that this age is less than the estimated cooling lifetime $\tau_{\text {cool }} \sim 12 \pm 2.3 \mathrm{Myr}$ for the $1.4 \mathrm{GHz}$ emitting CR electrons as they propagate through the ISM of 30 Dor. $^{13}$

Using this mean age of $3 \mathrm{Myr}$ in Equation (5), together with the best-fit Gaussian scale lengths corresponding to $l_{\text {diff }} \approx$ $140 \mathrm{pc}$ and $320 \mathrm{pc}$ for the $3 \mathrm{GeV}$ and $20 \mathrm{GeV}$ electrons and nuclei, respectively, we find a diffusion coefficient normalization constant of $D_{0} \approx(0.9-1.0) \times 10^{27} \mathrm{~cm}^{2} \mathrm{~s}^{-1}$. This is more than an order of magnitude lower than those found in the Milky Way (e.g., $\approx 5 \times 10^{28} \mathrm{~cm}^{2} \mathrm{~s}^{-1}$; Trotta et al. 2011). However, analytical solutions for the perpendicular diffusion coefficient (i.e., diffusion across magnetic field lines) are found to be much smaller. For example, Shalchi et al. (2010) report a perpendicular diffusion coefficient of $D_{\perp} \approx(3.0-30) \times 10^{26} \mathrm{~cm}^{2} \mathrm{~s}^{-1}$. Because 30 Dor is an active star-forming region, which likely has a strong turbulent magnetic field and lacks a large-scale regular field like the Galaxy, it may not be surprising that our derived value for the diffusion coefficient normalization factor is intermediate in the range for the perpendicular diffusion coefficients obtained by other authors.

\subsubsection{Results Including 3-10 GeV Maps}

So far we have only discussed the results comparing the propagation for $\sim 3 \mathrm{GeV}$ CR electrons and $\sim 20 \mathrm{GeV}$ CR nuclei. While it seems that the cooling time for the $1.4 \mathrm{GHz}(\sim 3 \mathrm{GeV})$ $\mathrm{CR}$ electrons is a factor of $\sim 4$ times larger than the average age of the stellar population in 30 Dor, trying to interpret the differences in their propagation lengths is complicated by the fact that the $\sim 3 \mathrm{GeV}$ CR electrons lose energy more rapidly than the $\sim 20 \mathrm{GeV}$ CR nuclei. That the electron energy losses are important is suggested by the result that the residuals between the $1.4 \mathrm{GHz}$ and $24 \mu \mathrm{m}$ maps are decreased more when using exponential kernels rather than Gaussian kernels. We therefore include the $3-10 \mathrm{GeV} \gamma$-ray map to provide an additional estimate for the propagation length of $\mathrm{CR}$ nuclei because it is sensitive to much higher energy CR nuclei (i.e., $\sim 70 \mathrm{GeV}$ for a proton energy index of 2.1). Employing these data, we can try to determine whether the difference between the CR electrons and protons is in fact the result of rigidity-dependent diffusion.

Applying the same smearing analysis to the $1.4 \mathrm{GHz}$ radio, 1-3 GeV, and 3-10 GeV $\gamma$-ray maps, but limiting the area of the residual calculation to that where there are $3-10 \mathrm{GeV}$ $\gamma$-ray events (i.e., $\approx 1 / 2$ the area having $1-3 \mathrm{GeV} \gamma$-ray events), we obtain the following results. The best-fit exponential and Gaussian scale lengths are 50 and 150 pc, respectively, for $\sim 3 \mathrm{GeV}$ electrons, 150 and $300 \mathrm{pc}$, respectively, for $\sim 20 \mathrm{GeV}$ CR protons, and 200 and $400 \mathrm{pc}$, respectively, for

\footnotetext{
13 This maximum lifetime is estimated using the combined energy losses due to synchrotron, inverse Compton, bremsstrahlung, and ionization processes (see, e.g., Murphy 2009). Using the previously estimated values for the minimum energy magnetic field strength $\left(B_{\min } \approx 11 \pm 4 \mu \mathrm{G}\right.$; see Section 2.2$)$, the radiation field energy density $\left(U_{\text {rad }} \approx 2.39 \pm 0.17 \times 10^{-12} \mathrm{erg} \mathrm{cm}^{-3}\right.$; see Section 2.4), and assuming an average ISM density of $n_{\mathrm{ISM}} \approx 2 \pm 1 \mathrm{~cm}^{-3}$ (Kim et al. 2003), we estimate a cooling time for the observed $1.4 \mathrm{GHz}$ emitting $(\sim 3 \mathrm{GeV})$ electrons of $\sim 12 \pm 2.3 \mathrm{Myr}$. We note that the individual lifetimes estimated against synchrotron, inverse Compton, bremsstrahlung, and ionization losses are $33,55,43$, and $140 \mathrm{Myr}$, respectively.
}

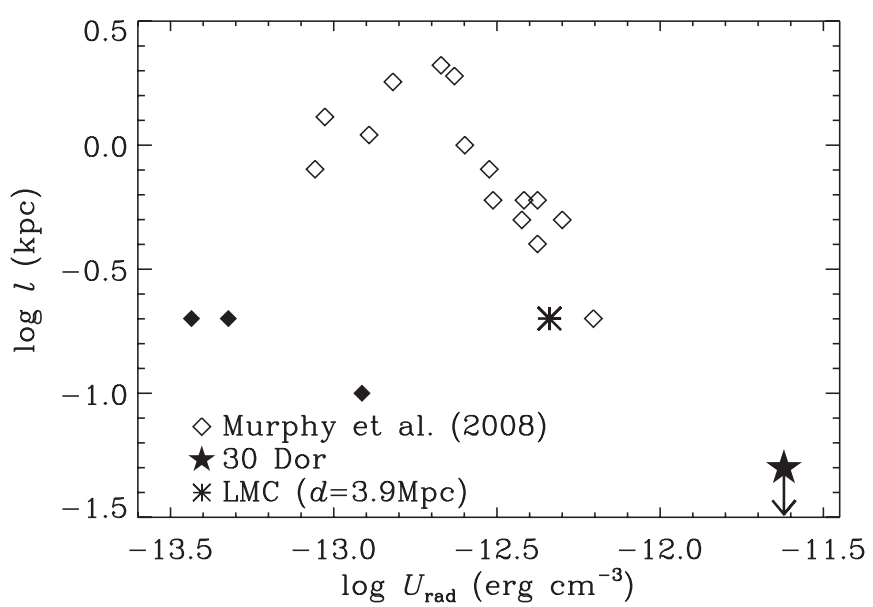

Figure 5. Best-fit exponential scale lengths plotted against radiation field energy density (measured by the infrared surface brightness) for the galaxies studied in Murphy et al. (2008, diamonds), the LMC (asterisk), and 30 Dor (filled star). The open diamonds indicate spirals, while the filled diamonds show the location of dwarf irregular galaxies included in the Murphy et al. (2008) study. The location of 30 Dor (filled star) follows the trend of decreasing exponential scale length, indicating shorter propagation lengths of CR electrons with increasing radiation field energy density for the more active star-forming disk galaxies in the Murphy et al. (2008) study. We plot the CR electron propagation distance for 30 Dor as an upper limit (see Section 4.2.3.) For the dwarf irregular galaxies, the short scale lengths are thought to be due to an increase in the escape of the CR electrons from the systems. If we set the LMC at the mean distance of the Murphy et al. (2008) dwarf irregulars (i.e., 3.9 Mpc), its propagation scale length is more consistent with the Murphy et al. (2008) spirals.

$\sim 70 \mathrm{GeV}$ protons. Because the detected $3-10 \mathrm{GeV}$ events are concentrated around the center of 30 Dor, lacking a significant extended component as observed in the 1-3 $\gamma$-ray map, it is not surprising that the scale lengths obtained are generally smaller than those calculated from the residuals over a larger area.

Using all six possible combinations of these values to solve for $\delta$ results in a median and dispersion of $\delta \approx 0.66 \pm 0.23$. This is consistent with the energy dependence estimated above using the $1.4 \mathrm{GHz}$ radio and $1-3 \mathrm{GeV} \gamma$-ray maps. We note that the value of $\delta$ estimated by comparing only the $\gamma$-ray maps, which is independent of assumptions for the magnetic field strength and proton energy index, is $\approx 0.51$, whereas the median when only including the $1.4 \mathrm{GHz}$ maps is $\approx 0.79$, suggesting that the bestfit scale lengths for the CR electrons may be underestimated due to additional energy losses. This is consistent with exponential kernels working slightly better to tighten the correlation between the $1.4 \mathrm{GHz}$ and $24 \mu \mathrm{m}$ maps compared to Gaussian kernels. Additionally, the estimate for the value of $\delta$ using only the $\gamma$-ray maps is independent of assumptions for the magnetic field strength. However, we emphasize that the $3-10 \mathrm{GeV} \gamma$-ray map is statistically limited, having $\gtrsim 3$ times fewer events than the 1-3 GeV maps.

\subsubsection{Propagation Length versus Star Formation Activity}

Having estimated the average distance traveled by CR electrons for an individual star-forming region, 30 Dor, it is interesting to see how these results compare with similar estimates of CR electron propagation distances for entire galaxies. Murphy et al. (2006b, 2008) reported a correlation between the CR electron propagation distance and galaxy surface brightness such that CR electrons are found to travel shorter distances, on average, in galaxies having higher star formation activity.

In Figure 5, we plot the results from Murphy et al. (2008, i.e., the best-fit exponential scale lengths projected in the plane 


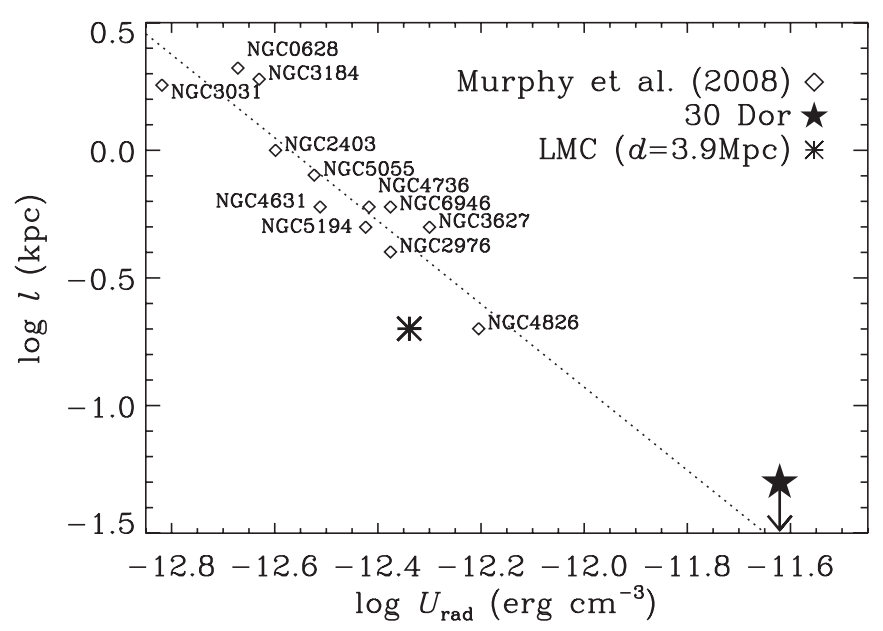

Figure 6. Same as Figure 5, except that we show only galaxies having high disk-averaged star formation rates. A distinct trend of decreasing CR electron propagation length with increasing radiation field energy density can be seen. Galaxies from the Murphy et al. (2008) study have been labeled. The dotted line is an ordinary least-squares fit only to the galaxies from Murphy et al. (2008). The locations of 30 Dor (filled star, as an upper limit) and the entire LMC (asterisk; scaled to $d=3.9 \mathrm{Mpc}$ ) are plotted.

of the sky versus $U_{\text {rad }}$ ) along with our estimates for 30 Dor. We note that various systematics and selection effects (e.g., distance, inclination) were rigorously investigated by Murphy et al. (2008). They additionally tested the differences between isotropic kernels and kernels projected in the plane of each galaxy disk, finding a preference, i.e., smaller residuals, for isotropic kernels.

The $U_{\text {rad }}$ estimate for 30 Dor was calculated from the surface brightness measured over an area of $2.4 \mathrm{kpc}^{2}$, the same region for which the residuals were estimated (see Section 2.4). For this case, the best-fit scale length was measured by comparing the free-free-corrected $1.4 \mathrm{GHz}$ with smoothed $70 \mu \mathrm{m}$ maps to allow a proper comparison with the results of Murphy et al. (2008). The best-fit exponential and Gaussian kernel scale lengths were 0 and $50 \mathrm{pc}$, respectively. Because Murphy et al. (2008) plot exponential kernel scale lengths, we plot the result from smoothing the $70 \mu \mathrm{m}$ map of 30 Dor with a Gaussian kernel as an upper limit.

Included in Figure 5 are both spirals and star-forming irregulars. The latter have markedly small best-fit scale lengths for their values of $U_{\text {rad }}$ relative to the sample of spirals. This is thought to be caused by increased escape of CR electrons in these systems (e.g., Cannon et al. 2005, 2006; Murphy et al. 2008). To see how the behavior of entire galaxies compares with the LMC as a whole, we repeat the image smearing analysis using the $70 \mu \mathrm{m}$ maps after first projecting the LMC to the mean distance of the Murphy et al. (2008) sample irregulars, $d=3.9 \mathrm{Mpc}$ to take possible resolution effects into account. We calculate the residuals and $U_{\text {rad }}$ within an aperture having a radius of $\approx 3.1 \mathrm{kpc}(2.75$ at the distance of $3.9 \mathrm{Mpc})$ centered at $05^{\mathrm{h}} 18^{\mathrm{m}} 50^{\mathrm{s}},-68^{\circ} 50^{\prime} 49^{\prime \prime}(\mathrm{J} 2000)$. This is approximately the area over which the $24 \mu \mathrm{m}$ emission was detected at $\gtrsim 3 \sigma$ level. The best-fit exponential and Gaussian scale lengths are 200 and $400 \mathrm{pc}$, respectively. This places the LMC close to the main trend among the star-forming spirals.

Focusing only on the region containing the star-forming spirals, we re-plot the disk galaxies along with 30 Dor and the LMC in Figure 6. The dotted line is a least-squares fit to the Murphy et al. (2008) galaxy disks, excluding the LMC and 30 Dor. Clearly, the position of 30 Dor in the plot is consistent with the empirical trend describing spiral galaxies, but extrapolated to a surface brightness greater by almost an order of magnitude. Thus, this scaling relation appears to operate on the scales of entire galaxies all the way down to individual star-forming regions.

\section{CONCLUSIONS}

Using a phenomenological image smearing model, we have estimated the typical propagation length of $\sim 3 \mathrm{GeV}$ CR electrons and $\sim 20 \mathrm{GeV}$ CR nuclei by comparing the spatial distributions of smoothed $24 \mu \mathrm{m}$ and (free-free-corrected) $1.4 \mathrm{GHz}$ and $1-3 \mathrm{GeV} \gamma$-ray maps, respectively. Below we list the major results and conclusions:

1. We estimate the typical distances traveled by $\sim 3 \mathrm{GeV} \mathrm{CR}$ electrons from 30 Dor to be $\sim 100-140 \mathrm{pc}$. This is factor of $\sim 2$ smaller than that estimated for $\sim 20 \mathrm{GeV}$ CR nuclei, which is found to be $\sim 200-320 \mathrm{pc}$.

2. In our image smearing analysis, we find that exponential kernels work slightly better to tighten the correlation between the $1.4 \mathrm{GHz}$ and $24 \mu \mathrm{m}$ maps compared to Gaussian kernels. In contrast, both exponential and Gaussian kernels are found to work equally well to tighten to correlation between the $1-3 \mathrm{GeV} \gamma$-ray and $24 \mu \mathrm{m}$ maps. This difference suggests that, unlike the CR nuclei, CR leptons may suffer additional energy losses or escape as they propagate through the ISM near 30 Dor on timescales less than, or comparable to, the diffusion timescale.

3. Assuming that the CR electrons and nuclei are produced by the same sources, and that their propagation is well described by a random walk, the differences in their estimated propagation lengths suggest differences in their associated spatial diffusion coefficients. This allows us to make the first model-independent measurement of the energy dependence of the diffusion coefficient for an external galaxy. For CRs produced in this star-forming region, the $\sim 20 \mathrm{GeV} \mathrm{CR}$ proton diffusion coefficient is $\sim 4$ times larger than that for $\sim 3 \mathrm{GeV}$ CR electrons, scaling as $(R / \mathrm{GV})^{\delta}$ where $\delta \approx 0.7-0.8$. This value is consistent with that obtained by including the more statistically limited $3-10 \mathrm{GeV} \gamma$-ray map $(\sim 70 \mathrm{GeV} \mathrm{CR}$ protons; $\delta \approx 0.66 \pm 0.23)$.

4. The value of $\delta$ reported here is larger than the spectral index of the diffusion coefficient assuming Kolomogorov turbulence and Milky Way secondary-to-primary ratios, and marginally consistent with Iroshnikov-Kraichnan turbulence. This may reflect the fact that 30 Dor region exhibits complex kinematic features, and fast expanding shells, resulting in a larger value of $\delta$.

5. Assuming that the CRs in 30 Dor are as old as the average stellar population $(\sim 3 \mathrm{Myr})$, we estimate a diffusion coefficient normalization constant of $D_{0} \approx 0.9-1.0 \times$ $10^{27} \mathrm{~cm}^{2} \mathrm{~s}^{-1}$. This value is less than and similar to modeldependent estimates of the parallel and perpendicular diffusion coefficient for the Galaxy, respectively. The similarity between our estimate and perpendicular diffusion coefficient values may be due the 30 Dor region having a magnetic field configuration that is highly turbulent.

6. We place our results for 30 Dor, and the LMC as a whole, in the context of the scaling relation between the typical CR electron propagation length and disk-averaged star formation activity for entire disk galaxies, where CR 
electron propagation is found to decrease with increasing star formation activity. This relation appears to apply to the LMC and the individual star-forming region of 30 Dor.

We thank the anonymous referee for useful suggestions that helped to improve the content and presentation of this paper. E. J. M. thanks Annie Hughes for both providing us with radio maps and for useful discussions which greatly helped shape the paper. We are grateful to the SAGE team for producing high quality data sets used in this study. T.A.P. and E.J.M. acknowledge support via NASA grant NNX10AE78G. I.V.M. acknowledges support via NASA grant NNX09AC15G.

The Fermi-LAT Collaboration acknowledges generous ongoing support from a number of agencies and institutes that have supported both the development and the operation of the LAT as well as scientific data analysis. These include the National Aeronautics and Space Administration and the Department of Energy in the United States, the Commissariat à l'Energie Atomique and the Centre National de la Recherche Scientifique/Institut National de Physique Nucléaire et de Physique des Particules in France, the Agenzia Spaziale Italiana and the Istituto Nazionale di Fisica Nucleare in Italy, the Ministry of Education, Culture, Sports, Science and Technology (MEXT), High Energy Accelerator Research Organization (KEK) and Japan Aerospace Exploration Agency (JAXA) in Japan, and the K. A. Wallenberg Foundation, the Swedish Research Council and the Swedish National Space Board in Sweden.

Additional support for science analysis during the operations phase is gratefully acknowledged from the Istituto Nazionale di Astrofisica in Italy and the Centre National d'Études Spatiales in France.

This work is based in part on observations made with the Spitzer Space Telescope, which is operated by the Jet Propulsion Laboratory, California Institute of Technology under a contract with NASA. This research has additionally made use of the NASA/IPAC Extragalactic Database (NED), which is also operated by the Jet Propulsion Laboratory, California Institute of Technology, under contract with NASA.

\section{REFERENCES}

Abdo, A. A., Ackermann, M., Ajello, M., et al. 2010a, A\&A, 523, L2 Abdo, A. A., Ackermann, M., Ajello, M., et al. 2010b, A\&A, 512, A7 Atwood, W. B., Abdo, A. A., Ackermann, M., et al. 2009, ApJ, 697, 1071 Beck, R., \& Krause, M. 2005, Astron. Nachr., 326, 414 Bell, E. F. 2003, ApJ, 586, 794

Bicay, M. D., \& Helou, G. 1990, ApJ, 362, 59

Boulares, A., \& Cox, D. P. 1990, ApJ, 365, 544

Calzetti, D., Kennicutt, R. C., Jr., Bianchi, L., et al. 2005, ApJ, 633, 871

Calzetti, D., Kennicutt, R. C., Engelbracht, C. W., et al. 2007, ApJ, 666, 870

Cannon, J. M., Smith, J.-D. T., Walter, F., et al. 2006, ApJ, 647, 293

Cannon, J. M., Walter, F., Bendo, G. J., et al. 2005, ApJ, 630, L37
Chu, Y.-H., \& Kennicutt, R. C., Jr. 1994, ApJ, 425, 720

Cox, D. P. 2005, ARA\&A, 43, 337

Crutcher, R. M. 1999, ApJ, 520, 706

Dale, D. A., \& Helou, G. 2002, ApJ, 576, 159

de Jong, T., Klein, U., Wielebinski, R., \& Wunderlich, E. 1985, A\&A, 147, L6 Dermer, C. D. 1986, A\&A, 157, 223

Ferrière, K. M. 2001, Rev. Mod. Phys., 73, 1031

Fields, B. D., Pavlidou, V., \& Prodanović, T. 2010, ApJ, 722, L199

Ginzburg, V. L., Khazan, I. M., \& Ptuskin, V. S. 1980, Ap\&SS, 68, 295

Harris, J., \& Zaritsky, D. 2009, AJ, 138, 1243

Haynes, R. F., Klein, U., Wayte, S. R., et al. 1991, A\&A, 252, 475

Helou, G., \& Bicay, M. D. 1993, ApJ, 415, 93

Helou, G., Roussel, H., Appleton, P., et al. 2004, ApJS, 154, 253

Helou, G., Soifer, B. T., \& Rowan-Robinson, M. 1985, ApJ, 298, L7

Hughes, A., Staveley-Smith, L., Kim, S., Wolleben, M., \& Filipović, M. 2007, MNRAS, 382, 543

Hughes, A., Wong, T., Ekers, R., et al. 2006, MNRAS, 370, 363

Iroshnikov, P. S. 1964, Sov. Astron., 7, 566

Kim, S., Staveley-Smith, L., Dopita, M. A., et al. 2003, ApJS, 148, 473

Klein, U., Wielebinski, R., Haynes, R. F., \& Malin, D. F. 1989, A\&A, 211 280

Kolmogorov, A. 1941, Akad. Nauk SSSR Dokl., 30, 301

Kraichnan, R. H. 1965, Phys. Fluids, 8, 1385

Lawton, B., Gordon, K. D., Babler, B., et al. 2010, ApJ, 716, 453

Lenain, J.-P., \& Walter, R. 2011, A\&A, 535, A19

Marsh, K. A., \& Helou, G. 1998, ApJ, 493, 121

Martínez-Galarza, J. R., Groves, B., Brandl, B., et al. 2011, ApJ, 738, 176

Marx, M., Dickey, J. M., \& Mebold, U. 1997, A\&AS, 126, 325

Meixner, M., Gordon, K. D., Indebetouw, R., et al. 2006, AJ, 132, 2268

Moskalenko, I. V., \& Strong, A. W. 1998, ApJ, 493, 694

Mountfort, P. I., Jonas, J. L., de Jager, G., \& Baart, E. E. 1987, MNRAS, 226 917

Murphy, E. J. 2009, ApJ, 706, 482

Murphy, E. J., Braun, R., Helou, G., et al. 2006a, ApJ, 638, 157

Murphy, E. J., Condon, J. J., Schinnerer, E., et al. 2011, ApJ, 737, 67

Murphy, E. J., Helou, G., Braun, R., et al. 2006b, ApJ, 651, L111

Murphy, E. J., Helou, G., Kenney, J. D. P., Armus, L., \& Braun, R. 2008, ApJ, 678,828

Nolan, P. L., et al. 2012, ApJS, in press (arXiv:1108.1435)

Niklas, S., \& Beck, R. 1997, A\&A, 320, 54

Nikolaev, S., Drake, A. J., Keller, S. C., et al. 2004, ApJ, 601, 260

Papadopoulos, P. P. 2010, ApJ, 720, 226

Papadopoulos, P. P., Thi, W.-F., Miniati, F., \& Viti, S. 2011, MNRAS, 414, 1705

Pavlidou, V., \& Fields, B. D. 2001, ApJ, 558, 63

Pérez-González, P. G., Kennicutt, R. C., Jr., Gordon, K. D., et al. 2006, ApJ, 648, 987

Pollack, J. B., \& Fazio, G. G. 1963, Phys. Rev., 131, 2684

Porter, T. A., Moskalenko, I. V., Strong, A. W., Orlando, E., \& Bouchet, L. 2008, ApJ, 682, 400

Ruzmaikin, A., Sokolov, D., \& Shukurov, A. 1988, Nature, 336, 341

Shalchi, A., Büsching, I., Lazarian, A., \& Schlickeiser, R. 2010, ApJ, 725, 2117

Socrates, A., Davis, S. W., \& Ramirez-Ruiz, E. 2008, ApJ, 687, 202

Staveley-Smith, L., Kim, S., Calabretta, M. R., Haynes, R. F., \& Kesteven, M. J. 2003, MNRAS, 339, 87

Strong, A. W., Moskalenko, I. V., \& Ptuskin, V. S. 2007, Annu. Rev. Nucl. Part. Sci., 57, 285

Strong, A. W., Porter, T. A., Digel, S. W., et al. 2010, ApJ, 722, L58

Tabatabaei, F. S., Beck, R., Krügel, E., et al. 2007, A\&A, 475, 133

Trotta, R., Jóhannesson, G., Moskalenko, I. V., et al. 2011, ApJ, 729, 106

Walborn, N. R., \& Blades, J. C. 1997, ApJS, 112, 457 